\title{
SENSOR PROPERTIES OF STABILIZED ZIRCONIA CERAMICS, MANUFACTURED FROM NANOPOWDERS
}

\author{
O.Yu. Kurapova ${ }^{1,2}$, M.M. Pivovarov'2, K. V. Nikiforova' ${ }^{1}$ and V.G. Konakovi,2
}

${ }^{1}$ St. Petersburg State University, Universitetskaya emb. 7/9, St. Petersburg, 199034, Russia

${ }^{2}$ Glass and Ceramics Ltd., 9 Linia V.O., 20, St. Petersburg, 199004, Russia

Received: April 26, 2018

\begin{abstract}
The paper reports the data on the sensing properties of $x \mathrm{CaO}-(100-x) \mathrm{ZrO}_{2}(\mathrm{CSZ}, x=9$, 12 and $15 \mathrm{~mol} . \%$ ) and $8 \mathrm{Y}_{2} \mathrm{O}_{3}-92 \mathrm{ZrO}_{2}$ (YSZ) solid electrolytes, manufactured from nanosized lowagglomerated powder. Stabilized zirconia solid electrolytes (SE) were synthesized via the original co-precipitation technique and annealing at $1823 \mathrm{~K}$ in air for 3 hours. Phase composition and conductivity of obtained ceramics was investigated via XRD and impedance spectroscopy techniques. Electromotive force (EMF) response on $\mathrm{O}_{2}$ partial pressure was obtained at $966 \mathrm{~K}$ for sensors with stabilized zirconia membranes. The data obtained was compared with Nernst equation. The impact of the precursor powder synthesis technique on electrical and sensing properties was discussed.
\end{abstract}

\section{INTRODUCTION}

Due to high conductivity, zirconia based ceramics is widely used as solid electrolyte membrane in various high temperature electrochemical devices, especially oxygen sensors [1,2]. Solid state oxygen sensors are necessary for the efficiency control of the various industrial processes where oxygen is implied [3], i.e. chemical, steelmaking, ferrous and nonferrous metallurgy, glass-making, for fuel combustion completeness monitoring in the car engines, etc. Despite number of binary systems fit the condition of high ionic conductivity, only $\mathrm{Y}_{2} \mathrm{O}_{3}$ $\mathrm{ZrO}_{2}$ (YSZ) based solid electrolytes have been successfully applied in industry. In fact, this sensor is a galvanic cell with zirconia based solid electrolyte (SE) with platinum or other noble electrodes applied from the both sides of the SE. The electromotive force (EMF) is formed due to the difference in the oxygen partial pressure on the working and reference electrode (typically, air oxygen). The working characteristics as well as performance of oxygen sensors during their exploitation in the heated haz- ardous gaseous atmosphere or a melt depend significantly on the phase stability, electrical, and mechanical properties of zirconia based SE. So the intensive work is performed nowadays to search the alternative solid electrolytes compositions as well as cost-effective production of ceramics with enhanced electrical and sensing properties. Calcia stabilized zirconia is known for decades for its high ionic conductivity [4-7]. Several problems were summarized by authors that limit its application as an oxygen sensor, like uniaxial compaction, low phase stability (tendency to recrystallization), high dopant volatility at the elevated temperatures. It is studied intensively as a model system for novel synthesis techniques development [8-10]. Calcia stabilized zirconia were obtained by citrate [11], rapid combustion techniques [12], various sol-gel approaches $[9,13]$. Their structure and electrical properties were investigated in details. The effect of ceramics composition and microstructure of the sintered pellets on the oxygen sensing characteristics was discussed in [13]. The EMF deviation from the theo-

Corresponding author: O.Yu. Kurapova, e-mail: olga.yu.kurapova@gmail.com 
Table 1. Numeration of zirconia solid electrolytes.

\begin{tabular}{|c|c|c|c|c|}
\hline Sample number & 1 & 2 & 3 & 4 \\
\hline Composition & $\begin{array}{l}9 \mathrm{CaO}-91 \mathrm{ZrO}_{2} \\
9 \mathrm{CSZ}\end{array}$ & $\begin{array}{l}12 \mathrm{CaO}-88 \mathrm{ZrO}_{2} \\
12 \mathrm{CSZ}\end{array}$ & $\begin{array}{l}15 \mathrm{CaO}-85 \mathrm{ZrO}_{2} \\
15 \mathrm{CSZ}\end{array}$ & $\begin{array}{l}8 \mathrm{Y}_{2} \mathrm{O}_{3}-92 \mathrm{ZrO}_{2} \\
\mathrm{YSZ}\end{array}$ \\
\hline
\end{tabular}

retical value of the CSZ sensor cell with $22 \mathrm{~mol} . \%$ $\mathrm{CaO}$ was larger than that of the CSZ sensor cell with $15 \mathrm{~mol} . \% \mathrm{CaO}$. The fact was explained from the conductivity position. The ionic transfer number of calcia stabilized zirconia (CSZ) thin film has been investigated in [14] using EMF method at low temperatures (573-673K) and low oxygen partial pressure. Surprisingly high sensitivity and quick response of sensors were obtained. Among the techniques mentioned, sol-gel synthesis was proved to be beneficial for solid electrolytes manufacturing. In the recent works of authors $[9,15]$ ceramic calcia stabilized SE with refined microstructure and high electrical properties were obtained by sol-gel synthesis in a version of the reverse co-precipitation with following freeze-drying. So, the goal of the present work was the investigation of the sensor properties of CSZ ceramic electrolytes by EMF technique.

\section{EXPERIMENTAL}

\subsection{Solid electrolytes synthesis}

Nanosized xCaO-(100-x) $\mathrm{ZrO}_{2}(\mathrm{CSZ}, \mathrm{x}=9,12,15$ mol.\%) and $8 \mathrm{Y}_{2} \mathrm{O}_{3}-92 \mathrm{ZrO}_{2}$ (YSZ) precursor powders were obtained by reversed co-precipitation from diluted aqueous salt solution. Washed CSZ gels underwent freeze-drying according to the technique, described in [15], whereas YSZ gels were dried under exceeding pressure [16]. Then the powders were annealed at $923 \mathrm{~K}$ for 3 hours in air. The obtained precursors were milled in a planetary mill (Pulverisette 6, Fritzch, 400 rpm, 1 hour) and compacted into the pellets (diameter $8.4 \mathrm{~mm}$, height 6 $\mathrm{mm}$, pressure 3.5 ton $/ \mathrm{sm}^{2}$ for CSZ and 5 ton $/ \mathrm{sm}^{2}$ for YSZ. Then the pellets were annealed at $1873 \mathrm{~K}$ for 3 hours. The pellets of $12 \mathrm{CaO}-88 \mathrm{ZrO}_{2}$ and $15 \mathrm{CaO}-$ $85 \mathrm{ZrO}_{2}$ (mol.\%) underwent intermediate microstructure refinement step to prevent recrystallization of ceramics according to [15]. Thus, four solid electrolytes were obtained, see Table 1.

\subsection{Analysis}

Phase composition of ceramics was identified using X-ray diffraction analysis (XRD, Shimadzu XRD6000 , Cu-Ka radiation, $\lambda=1.54 \AA$, room tempera- ture). Powder diffraction data was used for phase composition identification [17]. Electrochemical impedance (Autolab PGSTAT 302N Potentiosytat/ Galvanostat) was used to investigate the electrochemical properties of calcia stabilized zirconia solid electrolytes. Electrochemical impedance was measured in the temperature range from 523 to $1078 \mathrm{~K}$. Experiments were performed in the quartz cell with Pt electrodes and contacts.

\subsection{Galvanic cell, measurement conditions, and reproducibility}

In order to obtain the data on the oxygen sensor response with zirconia solid electrolytes, galvanic cell 1 (GC) was investigated at 966 and $976 \mathrm{~K}$

$\left(\mathrm{O}_{2}\right.$, air) Pt|zirconia SE $\operatorname{Pt}\left(\mathrm{O}_{2}, \mathrm{~N}_{2}\right) \quad$ (GC 1)

Platinum electrodes were braised from the paste to the both sides of the membrane and then baked at 1723K. Platinum current conductor in the form of a ring was placed in the interface of the SE and electrode. A number of oxygen - gry nitrogen mixtures was used for the measurements, the accuracy of gas mixture preparation was $\pm 5 \%$. For all galvanic cells tested, the total pressure was kept constant, $101 \mathrm{kPa}$. The temperature was measured by Pt/Pt$10 \% \mathrm{Rh}$ thermocouple. The cell electromotive force (EMF) measurement was carried out with $\pm 0.1 \mathrm{mV}$ accuracy.

\section{RESULTS AND DISCUSSION}

Typical XRD patterns obtained for YSZ and CSZ solid electrolytes are shown in Fig.1.

As it can be seen from the figure, phase composition of YSZ SE corresponds to well-formed cubic zirconia based solid solution without any admixtures of the other phases. Indeed, the crystallinity degree of sample 4 is close to theoretical, i.e. $97 \%$ (see Table 2). At the same time, sample 1 consists of two phases - cubic zirconia based solid solution and monoclinic zirconia (baddeleite), indicating the recrystallization. Similar results were obtained for all calcia stabilized zirconia SE studied. Cubic solid solution is the main phase in all cases. The amount 

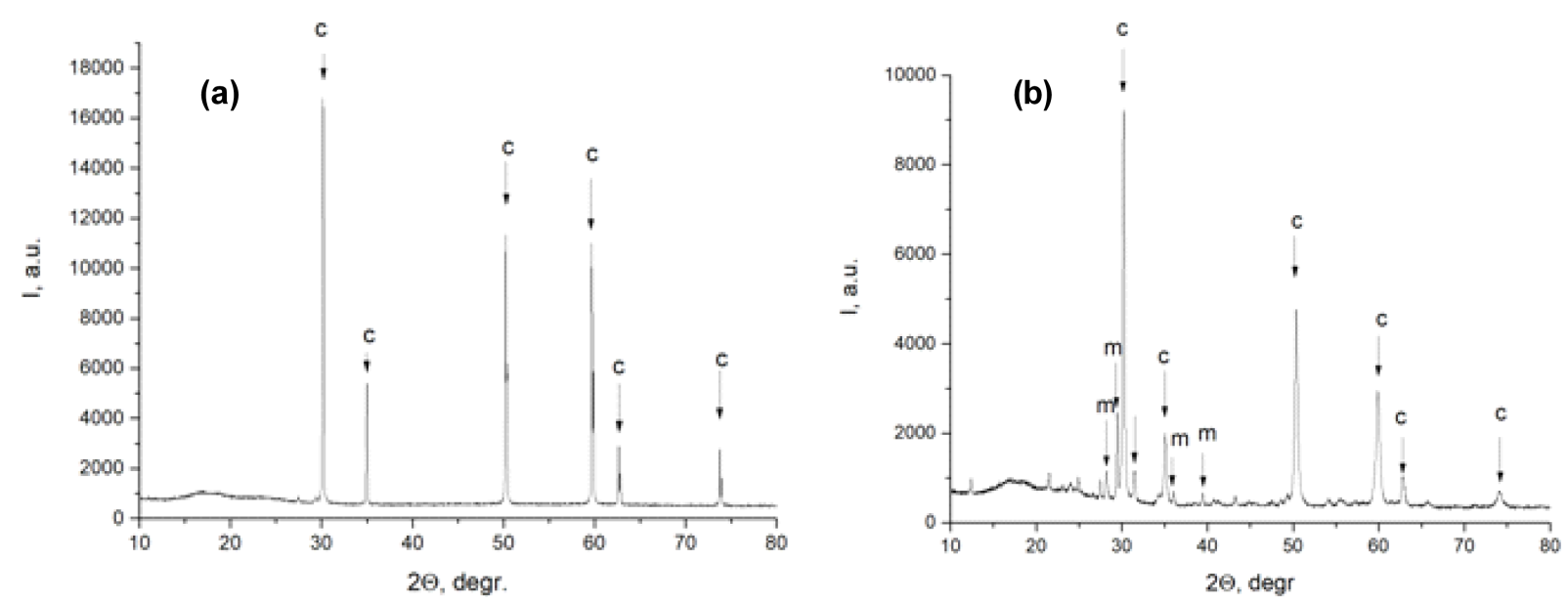

Fig.1. XRD patterns of sample (a) 4 - YSZ solid electrolyte and (b) 1 - 9CSZ solid electrolyte, c - cubic solid solution, $\mathrm{m}$ - monoclinic zirconia (baddeleite).
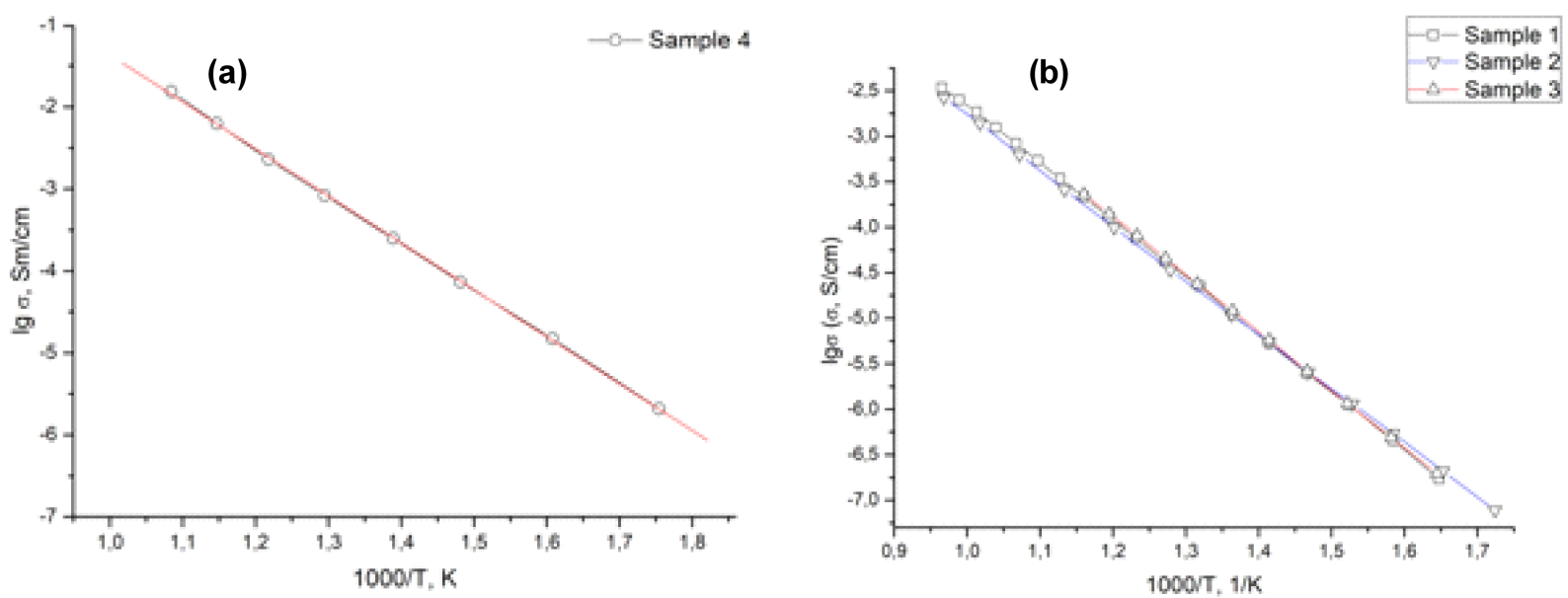

Fig. 2. Temperature dependencies of the integral conductivity obtained using impedance data for samples (a) 4 and (b) 1-3.

of baddeleite varies depending on the composition from 7 to $15 \%$ (see Table 2 ).

It should be noted that the size of the pellets manufactured for oxygen sensitivity tests is rather small; the diameter of the pellet is comparable with its height. The recrystallization processes observed are, most likely, due to the intensive evaporation of calcia during the calcination at $1823 \mathrm{~K}$.

The data on SE conductivity were obtained using impedance technique. The detailed analysis of impedance spectra of calcia stabilized zirconia SE was performed by authors in $[9,15]$. It was shown earlier that impedance spectra for each sample consist of two distinguished semi-circles correspond- ing to ionic conductivity, see [9] for details.. Temperature dependencies of the integral conductivity obtained using impedance data for samples 1-4 are presented in Fig. 2.

Linear dependence of integral conductivity on temperature is observed for all zirconia based SE in the range $556-1000 \mathrm{~K}$. Conductivity is ionic with no admixture of electronic component. The conductivity values of samples $1-3$ containing 9,12 , and 15 mol. $\% \mathrm{CaO}$, respectively, are rather close to each other in all temperature range. It is, probably, due to similar SE phase composition. Conductivity value of calcia stabilized zirconia SE is $\sim 10^{-2} \mathrm{Sm} / \mathrm{cm}$ at $1000 \mathrm{~K}$ (see Fig. 2b) whereas yttria stabilized

Table 2. Phase composition and crystallinity degree of zirconia based SE.

\begin{tabular}{lllll}
\hline Sample number & 1 & 2 & 3 & 4 \\
\hline Phase composition & $\mathrm{C}+\mathrm{M} \sim 93: 7 \%$ & $\mathrm{C}+\mathrm{M} \sim 85: 15 \%$ & $\mathrm{C}+\mathrm{M} \sim 80: 10 \%$ & $\mathrm{C}, 100 \%$ \\
Crystallinity & $82 \%$ & $87 \%$ & $89 \%$ & $97 \%$ \\
\hline
\end{tabular}



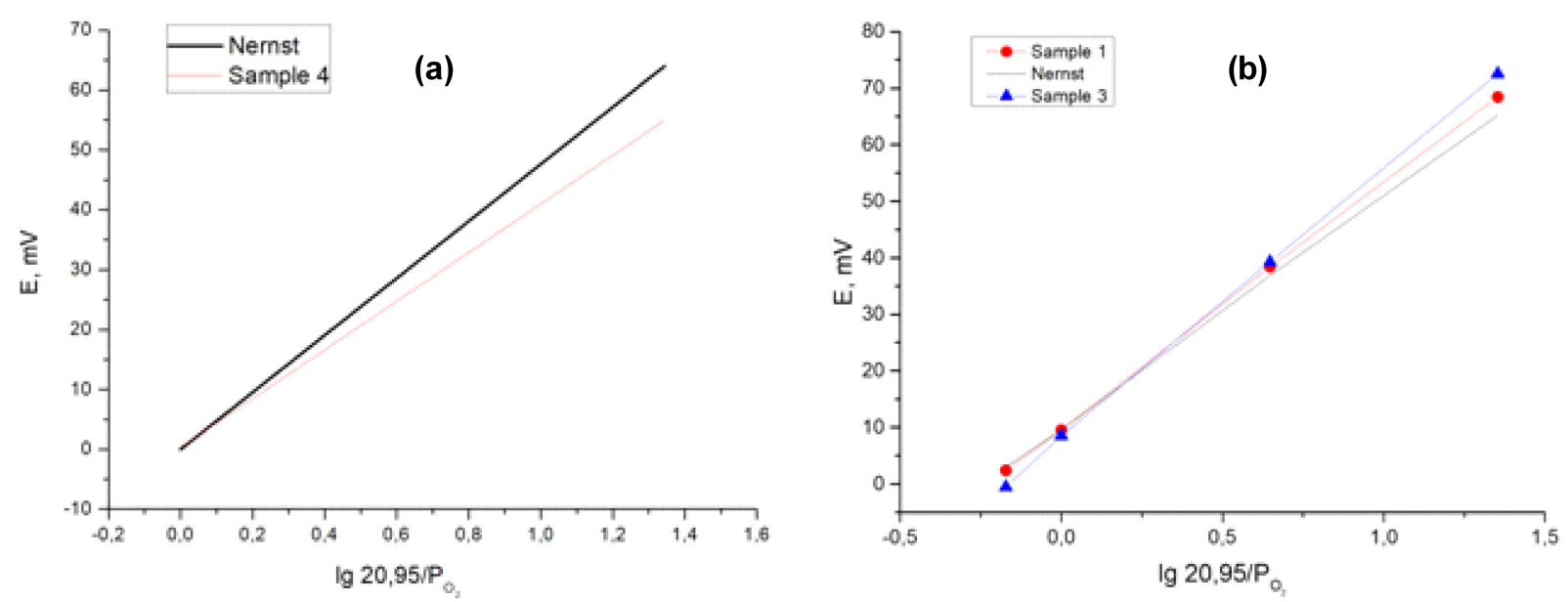

Fig. 3. EMF dependence on the relative oxygen partial pressure for GC1 with (a) YSZ membrane (sample 4 ) at $960 \mathrm{~K}$ and (b) $9 \mathrm{CSZ}$ and $15 \mathrm{CSZ}$ membranes (samples 1 and 3) at $976 \mathrm{~K}$.

zirconia shows one order of magnitude higher conductivity values i.e. $\sim 10^{-1} \mathrm{Sm} / \mathrm{cm}$ at $1000 \mathrm{~K}$.

Then the data about oxygen partial pressure response of the sensors with zirconia based SE were obtained by EMF method. In order to get fast response, the experiment was performed at temperatures close to $1000 \mathrm{~K}$. Following reactions are taking place on the electrodes:

$\mathrm{O}_{2}+4 \mathrm{e} \rightarrow 2 \mathrm{O}^{2-}$,

$2 \mathrm{O}^{2-} \rightarrow \mathrm{O}_{2}+4 \mathrm{e}$.

So, the EMF of GC 1 can be described as follows:

$E=\left(\theta \lg p\left(\mathrm{O}_{2}\right) / p_{\text {ret }}\left(\mathrm{O}_{2}\right)\right) / 4$,

where $\theta=2.3 R T / F$ and $p_{\text {ref }}\left(\mathrm{O}_{2}\right)=20.95$ vol.\% is air oxygen.

The EMF dependence of sensors with zirconia membranes on the relative oxygen partial pressure is presented in Fig. 3. The experimental data was compared with Nernst EMF dependence. It should be noted that sensor with $12 \mathrm{CSZ}$ membrane did not show the response to oxygen partial pressure, which is probably due to the high content of monoclinic phase admixture.

As it is seen from Fig. 3, linear EMF dependence on temperature is observed for sensors with both YSZ and CSZ SE in all investigated oxygen partial pressure range. For calcia stabilized zirconia sensors obtained using cryochemical technique the deviation from the Nernst equation is less than 5-7 $\mathrm{mV}$ at the lowest oxygen partial pressure $\left(\mathrm{p}\left(\mathrm{O}_{2}\right)=\right.$ 0.928 vol.\%). The data obtained for the sensors with $9 \mathrm{CSZ}$ and $12 \mathrm{CSZ}$ in the oxygen partial pressure range from 4.72 to 31.14 vol.\% are in agreement with the theoretical data. In case of sensor with YSZ membrane obtained using drying under exceeding pressure, the EMF deviation from Nernst equation is slightly higher comparing to CSZ SE. At the lowest partial pressure this value is $10 \mathrm{mV}$, which is still within the error of the high temperature measurement. Despite higher integral conductivity of YSZ ceramic SE (see Fig. 2), the sensors with calcia stabilized zirconia SE showed the best Nernst correspondence. Comparing the results of the present work with data of [13] for sensors with 15CSZ SE, one can see that matching to the theoretical value is much better in case of ceramics manufactured by chryochemical approach. Authors of [13] suggest that the difference in sensor response for 15 and $22 \mathrm{~mol} . \%$ of calcia stabilized zirconia is due to the conductivity level. However, from Figs. 2 and 3 it is seen that YSZ has one order of magnitude higher conductivity values, while the deviation from the theoretical value for such sensor is greater. The observed difference is, most likely, due to the refined microstructure of calcia-stabilized zirconia solid electrolytes obtained by cryochemical technique. Indeed, as it was shown by authors recently [15], the use of reversed co-precipitation technique with following freeze-drying and intermediate thermal treatment of ceramics results in dense ceramic solid electrolytes characterized by the refined microstructure with microsized grains of similar size divided by the welldefined grain boundaries. From Figs. 1-3 it is seen that the presence of $\sim 7-10 \%$ of monoclinic phase admixture do not result in sensor properties decrease, however, SE sensor loses the response to oxygen when the baddeleite contents reaches $15 \%$ as it tooks place for $12 \mathrm{CSZ}$ ceramics. Note, that yttria stabilized zirconia was prepared from nanosized powder of the same mean size as CSZ precursors using co-precipitation and drying under exceeding pressure, which is, not that efficient for 
the agglomeration elimination at the drying step. Thus, the correct choice of the precursor powders synthesis technique predetermines the electrical and sensor properties of solid electrolytes due to the possibility of microstructure control on each synthesis step.

\section{CONCLUSIONS}

Via XRD and impedance spectroscopy it was shown that cryochemical approach is an effective and scalable way to manufacture cubic zirconia solid solution electrolytes having conductivity of $\sim 10^{-1}-10^{-2} \mathrm{Sm} /$ $\mathrm{cm}$ at $1000 \mathrm{~K}$. Sensors with YSZ, 9CSZ and $15 \mathrm{CSZ}$ solid electolytes showed linear EMF response to relative oxygen partial pressure in all the investigated $p\left(\mathrm{O}_{2}\right)$ range. The deviation from the theoretical value is minimal in case of sensor with $9 \mathrm{CSZ}$ solid electrolyte.

\section{ACKNOWLEDGEMENTS}

The research work was supported by the special President's scholarship for young scientists (research project CP-1967.2016.1). The authors are grateful to Alexander Shorokhov for the valuable help with EMF data measurement.

\section{REFERENCES}

[1] V. V. Kharton, F. M. B. Marques and A. Atkinson // Solid State Ionics 174 (2004) 135.

[2] D. J. Fray // Solid State lonics 86-88 (1996) 1045.

[3] V.G. Konakov // Journal of Solid State Electrochemistry 15 (2011) 77.

[4] N. M. Beekmans, L. Heyne // Electrochimica Acta 21 (1976) 303.
[5] S. H. Chu and M. A. Seitz // Journal of Solid State Chemistry 23 (1978) 297.

[6] V. S. Stubican and P. S. Ray // Journal of the American Ceramic Society 60 (1977) 53.

[7] K. Wang, C. H. Li, Y. H. Gao, X. G. Lu and W. Z. Ding // J. Amer. Cer. Soc. 92 (2009) 1098.

[8] O. Yu. Kurapova, D. V. Nechaeva, A. V. Ivanov, S. N. Golubev, V. M. Ushakov and V. G. Konakov // Rev. Adv. Mater. Sci. 47 (2016) 96.

[9] O. Y. Kurapova, O. V. Glumov, M. M. Pivovarov, S. N. Golubev and V. G. Konakov // Reviews on Advanced Materials Science 52 (2017) 134.

[10] O. Yu. Kurapova, V. G. Konakov, S. N. Golubev, V. M. Ushakov and I. Yu. Archakov // Rev. Adv. Mater. Sci 32 (2012) 112.

[11] R. Muccillo, R. C. Buissa Netto and E. N. S. Muccillo // Mater. Letters 49 (2001) 197.

[11] A. K. Shukla, V. Sharmaa, N. Arul Dhas and K. S. Patil // Materials Science and Engineering: B 40 (1996) 153.

[13] M. Zhou and A. Aftab // Materials research bulletin 41 (2006) 690.

[14] M. Croset, P. Schnell, G., Velasco and J. Siejka // Journal of Vacuum Science and Technology 14 (1977) 777.

[15] O. Y. Kurapova, O. V. Glumov, I. V. Lomakin, S. N. Golubev, M. M. Pivovarov, J. V. Krivolapova and V.G. Konakov // Ceramics International 44 (2018) 15464.

[16] V.G. Konakov, S. Seal, E.N., Solovieva, D.A. Ivanov-Pavlov, M. M. Pivovarov, S. N. Golubev and A.V. Shorohov // Rev. Adv. Mater. Sci. 16 (2007) 96.

[17] PDF (powder diffraction file) database, release 2, 2012. 